\title{
Adding Social Media to E-Learning in the Workplace: Instilling Interactive Learning Culture
}

\author{
http://dx.doi.org/10.3991/ijac.v5i3.2183 \\ Juha Leino, Erika Tanhua-Piiroinen and Johanna Sommers-Piiroinen \\ The University of Tampere/School of Information Sciences/TRIM, Tampere, Finland
}

\begin{abstract}
As social media features are increasingly added to e-learning, we urgently need more case studies of their use to ground practices in actual experience rather than hype. Using ethnography-based approach, we studied five continuous professional development pilot trainings where learning largely took place at workplace through wikis, blogs, forums, chats and voice conferencing. Learners valued interactivity, peer support and abundant, instant feedback offered by synchronous features. Simultaneously, however, asynchronous features were often treated as chores and overall interactive learning culture failed to emerge. Instructors, while explicitly encouraging social learning approach, implicitly reinforced teacher-centered learning, leading learners to stick to conventional learning culture. Also, training designs often failed to engender interaction. Moreover, at general level, group sizes were too big, moderation was not used efficiently, and differing skill sets of learners were not evened out. The lessons learned from these trainings offer us insight into how to design elearning enhanced with social media and how to avoid potential pitfalls. Particularly, we discuss designing interactivity into the e-learning process.
\end{abstract}

Index Terms-E-learning, social media, workplace.

\section{INTRODUCTION}

Social media tools and approaches, based on Web 2.0 paradigm, offer educational affordances in that they can support social interaction, e.g. sharing, collaborating and communicating $[1,2,3]$. Social tools, e.g. wikis, chats and blogs, are seen as a way of integrating social learning into e-learning in both constructivist and collaborative sense $[1,4]$. As peer interaction in social context is considered to lead to effective construction of meaning and to greater learning than teacher-oriented instruction and memorization [1, 4, 5, 6], "sociability aspects" afforded by social tools are seen as having "the most potential for enhancing education" [3]. Using such tools also fits in with the current initiatives to move to more student-centered learning [6].

Consequently, social media features are increasingly used in formal education and are also making inroads to continuous professional development (CPD) in the workplace $[2,7]$. Simultaneously, however, many case studies of using such tools have been less than successful (e.g. [1] and [8]). We do not know "why and under what conditions cooperative peer-based learning is effective" [5]. Much of the uncritical evangelizing of the educational potential of social software is still unsubstantiated [2] and there is a real danger of "misunderstandings, disappointments and irrelevant pedagogical practices" [9].

In effect, the whole field is in a dire need of case studies $[2,7,10]$, particularly "qualitative, student-centered work" [11], to uncover the actual dynamics emerging from using social tools in e-learning and to guide adding and using them [5, 7, 10]. Moreover, different perspectives in educational and workplace-related e-learning mean that results from one cannot necessarily be directly applied to another $[10,12]$. Consequently, we need case studies in the field of CPD urgently, especially since it has been studied even to a lesser degree than formal education $[5,7,10]$.

Our study affords an in-depth look at the actual dynamics of what happens when social media tools and approaches are incorporated into e-learning in the workplace context. We studied five pilot trainings provided by two professional training organizations in co-operation. All trainings were planned to be largely carried out with social media tools although all also had at least two contact teaching days. The organizer and the client organizations were all major Finnish companies in their respective fields.

We studied the trainings from two viewpoints: through perceptions and experiences of learners and through those of instructors. While learner experiences are obviously essential, instructor perceptions are similarly important. Instructors determine how social media is incorporated into e-learning and the requirements instructors set explicitly (e.g. verbal and written instructions, assignments, and comments) and implicitly (e.g. by behavior or tone of voice) frame and influence learner behavior and experience.

In this article, we discuss the implications of the learner and instructor experiences, focusing on what worked, what did not, and how to instill interactive learning culture. Despite many challenges, our results largely support claims about the potential of social media tools to enhance learning. Peer interaction provided value to learners in form of ideas and suggestions, experiencebased feedback and peer support. However, numerous challenges, especially concerning asynchronous tools, need to be addressed before the full potential of social media is unleashed. Benefits are not automatic, and these kinds of trainings require careful designing and maintaining.

\section{BACKGROUND}

Although social media tools are not new per se, the way they will be used for learning will be new in comparison 
to their other use contexts [3]. We need to understand better the educational affordances of the tools and how to engender knowledge-creating process with them $[5,6]$. Adding social media features is no guarantee that they are used properly-or at all-or that using them leads to learning $[1,3,5,9]$.

Furthermore, learning with social media tools and approaches is not simply a question of "an assembly of tools, software and digital strategies, but [of] a set of concepts, practices and attitudes" [3] that need to be "invented and designed" [12].Without both learners and instructors internalizing the new approach and its practices and attitudes, all-important mindset change might fail to take place and necessary behavioral changes might not materialize [3, 12].

Instructors need to communicate the new approach to learners who may otherwise fail to see interactive, social e-learning as real learning because of it not fitting their pre-conceptions of learning [11]. Benefits of the new approach need to be explicated to manage "student expectations and behaviour in relation to activities that lack a directly assessed component" [1]. When students are given "a valid pedagogical reason for engagement," they engage better with the learning environment [13].

In addition, as we are moving from teacher-centered instruction to learner-centered learning [6], instructors need to appreciate that their role is also changing from teaching to advising, facilitating and motivating [8, 10]. The information no longer flows from expert(s) to learners-knowledge is constructed through discussion and exploration, and instructors need to mediate and facilitate this process $[8,14]$.

However, as instructors still retain their evaluative powers, the exact role division is likely to continue to baffle both sides for some time to come [2]. Learners often expect instructors to participate more actively and to provide more corrective feedback than instructors feel is appropriate in the bottom-up pedagogy of social elearning [2]. Experience leads to better facilitation [4], as striking the balance between over-participation (that stifles conversation) and under-participation (that leaves learners to question the meaningfulness of the activity) is challenging $[2,14]$.

Likewise, learners need to adopt a more active and participatory role $[3,10]$. Learners become prosumers, i.e. both producers and consumers of content and knowledge, instead of passive consumers [1, 3]. However, as students prioritize their time by perceived benefit, anything that lacks a directly assessed component receives less attention $[1,5]$. There is also the problem of lurkers or social loafers: Not everybody contributes equally, and those who contribute can be frustrated by the lack of input from others $[5,14]$.

Since today "education exists in a consumerist culture where altruistic acts are devalued and individual effort is rewarded" and "perceived usefulness is directly related to assessment structures" [1], we need to re-consider how to assess learners and how learning becomes visible. If interactive and participatory process is at the heart of social e-learning, then we need to assess learners based on it $[1,13]$.

\section{METHODOLOGY AND DATA USED}

Our research goal was to understand how to successfully incorporate social media tools into CPD trainingswhat factors were crucial for success and what pitfalls there were and how to avoid them. As studying complex social phenomena taking place in social e-learning necessitates qualitative case study approach [7], we used an ethnography-based approach in studying five pilot trainings where formal workplace trainings previously taught through contact teaching were now largely taught through social media tools. Table 1 summarizes the trainings by type $(\mathrm{O}=$ open vs. $\mathrm{T}=$ tailored $)$, field $(\mathrm{R}=$ retail vs. $\mathrm{I}=$ insurance/finance), social media tools used, number of learners, and instructors involved.

TABLE I. PILOT TRAINING SUMMARY.

\begin{tabular}{|c|c|c|c|c|c|}
\hline & T1 & T2 & T3 & T4 & T5 \\
\hline Type & $\mathrm{T}$ & $\mathrm{T}$ & $\mathrm{O}$ & $\mathrm{T}$ & $\mathrm{T}$ \\
\hline No. of learners & 13 & 9 & 14 & 40 & 15 \\
\hline Instructors & $\begin{array}{l}\mathrm{I} 1, \\
\mathrm{I} 4\end{array}$ & $\begin{array}{l}\mathrm{I} 1, \\
\mathrm{I} 4\end{array}$ & $\begin{array}{l}\text { I2, I4, } \\
\text { I5 }\end{array}$ & $\mathrm{I} 2, \mathrm{I} 4$ & I3, I4 \\
\hline Target field & $\mathrm{R}$ & $\mathrm{R}$ & I & $\mathrm{I}$ & $\mathrm{I}$ \\
\hline Length & $\mathrm{n} / \mathrm{a}$ & $\mathrm{n} / \mathrm{a}$ & 2 mths & 3 mths & $\begin{array}{c}15 \\
\text { mths }\end{array}$ \\
\hline $\begin{array}{r}\text { No. of contact } \\
\text { days }\end{array}$ & $\mathrm{n} / \mathrm{a}$ & $\mathrm{n} / \mathrm{a}$ & 2 & 2 & 8 \\
\hline Wiki & \multirow{5}{*}{\multicolumn{2}{|c|}{$\begin{array}{l}\text { Problems } \\
\text { prevented } \\
\text { the use of } \\
\text { social media } \\
\text { tools. }\end{array}$}} & & & $1^{\mathrm{a}}$ \\
\hline Blog & & & & $3^{b}$ & $1^{\mathrm{c}}$ \\
\hline Forum & & & 3 & 2 & 2 \\
\hline Chat & & & 3 & 3 & 1 \\
\hline Voice conf. & & & & 2 & \\
\hline
\end{tabular}

The pilot trainings were organized, designed and run by instructors hailing from two professional training organizations. Many instructors had multiple roles (course designers, moderators, trainers or technical facilitators). Some of the roles were intertwined. In addition to observing moderation in action (e.g. chats) and through artifacts (e.g. blog comments and assignment descriptions), we also interviewed four instructors at different points of the piloting process and received written reflections from three (Table 2). Having both interview and observation data is important to avoid the say-do problem.

TABLE II.

INSTRUCTORS BY INTERVIEWS AND REFLECTIONS.

\begin{tabular}{|l|l|l|l|l|l|}
\hline & $\mathrm{I} 1$ & $\mathrm{I} 2$ & $\mathrm{I} 3$ & $\mathrm{I} 4$ & $\mathrm{I5}$ \\
\hline Beginning interview (phone interview) & $\mathrm{X}$ & $\mathrm{X}$ & $\mathrm{X}$ & & \\
\hline Midpoint interview (phone interview) & & \multirow{2}{*}{$\mathrm{X}^{\mathrm{d}}$} & & $\mathrm{X}$ & \\
\cline { 1 - 3 } \cline { 6 - 8 } & $\mathrm{X}$ Ening interview (phone interview) & $\mathrm{X}$ & & & \\
\hline Ending reflections (written) & & $\mathrm{X}$ & & $\mathrm{X}$ & $\mathrm{X}$ \\
\hline
\end{tabular}

In the planning phase, before the trainings actually started, we wanted to understand how instructors saw enhancing such training with social media tools-what goals they had, what benefits they expected, what problems whey expected and how they prepared to solve those problems, and how these considerations affected the planning and designing of the training. Also, we asked

\footnotetext{
${ }^{\mathrm{a}}$ Each learner was involved in making 1-4 wiki pages in small groups.

${ }^{\mathrm{b}}$ One deadline but each learner had to write on three themes.

${ }^{\mathrm{c}}$ Continuous learning diary.

${ }^{\mathrm{d}}$ The two interviews were combined because the instructor left the training organization at this point.
} 
about their prior experiences of using social media tools in general and in particular for training/education. Instructors had mainly used Facebook and some had experiences of learning with social media tools (e.g. Moodle) but overall they did not have much experience beyond that of an average person. Only one instructor was technically familiar with social media tools, and he was the one responsible for building the actual learning environments (first with Ning and, later on, Elgg, in addition to B2Evolution (blogging) and DokuWiki).

During the training process we wanted to see how instructors behaved and approached various situations. Also, during and especially after trainings we wanted to hear how instructors had experienced each training, what had worked and what not in their opinion, and how they planned to develop and improve trainings in future based on their experiences.

When it comes to learners, we in fact focus on T3-T5 because in $\mathrm{T} 1$ and $\mathrm{T} 2$ learners never actually used social media tools due to technical problems (e.g. chats updating too slowly and firewalls preventing connections) in addition to a host of other problems. Consequently, T1 and T2 only serve to provide examples of pitfalls.

With learners, we used observing interactions (e.g. chats), online artifacts (e.g. blog texts) and log information (e.g. wiki history) in addition to collecting questionnaire data and interviewing learners, both individually (T4) and in groups (T5). Moreover, some forum, blog and chat topics were related to using social media tools in the training.

With learners, we likewise wanted to know what expectations and goals they had for social media enhanced trainings and how much prior experience they had with social media tools in general and with e-learning in particular. During the trainings we focused on observing how learners behaved. After the trainings, we wanted to understand what had motivated the observed behavior and how learners had experienced the training-what had worked for them and what had not, and how they would improve the trainings. We were interested in both larger contextual matters (e.g. what learning with social media tools meant in practice at workplace) and also in detailed experiences with individual tools (e.g. learner views on blogs).

We used a typical data coding approach to analyze the qualitative data. After transcribing non-text materials, we coded them by research themes that had also served as a foundation for the semi-structured interviews for learners and instructors. These themes were further coded into subcategories based on themes emerging from the material. Questionnaires also provided us with some quantitative data.

\section{LEARNER AND INSTRUCTOR PERCEPTIONS}

\section{A. Instructor expectations and views on outcomes}

Instructors expected adding social media tools to increase sociality and interaction among learners. They hoped that this would positively affect group cohesion and motivation in addition to spreading learning to periods between contact days, thus creating a continuous learning process and experience and integrating learning into the working day.

Instructors wished to move towards learner-centricity and hoped that using social tools would result in a multi- plicity of views and enrichment of the learning materials. Consequently, they aimed at learners adopting interactive, conversational style and contributing also opinions and smaller observations rather than submitting finalized assignments.

Furthermore, instructors felt that learner-centricity required learners to adopt more active roles and take responsibility for their own learning, while instructors also needed to give up their authority position to make space for collective formulation of knowledge and to allow the knowledge of learners to emerge. However, simultaneously, instructors saw a danger in leaving too much up to learner initiative and felt that they needed to encourage activity to avoid turning the social media platform into " $a$ desert of silence." Instructors considered behavioral example and encouragement their main tools in instilling the new learning culture on learners.

When looking at the outcome, however, instructors felt that they had largely failed to replace the old, school-like learning culture with a new social one. Postings ended up "very much thought-out," lacking conversational style and informality they had sought to increase; e.g. one instructor described forum postings as "series of traditional monologues." Nevertheless, instructors felt that "some peer interaction took place” and grouping was improved.

However, instructors were not discouraged, as they felt that integrating social media tools to CPD trainings was a process and getting it right the first time was unlikely anyway: "I learned that in teaching I need to learn to more and more ask good questions and stay quiet instead of bringing forth my own views..." Also, instructors felt that they had to integrate the use of various tools into a more continuous whole and use more compulsoriness to generate commenting. Finally, they felt that they had to explicate the benefits and the process of the new approach more clearly.

\section{B. How learners perceived their training}

Overall, trainings were viewed in a moderately positive light. In the end-survey of T4 $(n=21)$, the learner satisfaction (1=Very unsatisfied; $7=$ Very satisfied) with the training was 4.6 (STDEV 1.1). When asked if social media tools helped in learning, the result was a noncommittal 4.33 (STDEV 1.8). As the STDEV indicates, views were widely dispersed. The satisfaction ratings were negatively correlated $(-0.6 ; \mathrm{p}<0.000)$ with the ratings of whether social media tools were used too much in the training (4.0; STDEV 2.0); lack of satisfaction went handin-hand with the perception of too much social tool use. In fact, the learners who felt that peer-sharing of experiences represented real learning saw their training positively_- "It feels great to ... discuss and to share thoughts with people who work in the same field"-while those who did not, saw it as lacking: “...it should have been more professional. ...we were just sharing our own experiences. ... Although it's fun and all, there should have been some concrete teaching, too."

Based on interviews, $\mathrm{T} 5$ viewed the training more positively than T4 overall but at the same time had little liking for the social media tools that they largely felt had been "glued on top." What they in fact liked were the contact days. Likewise, T4 expressed a strong liking for contact days at 6.3 (STDEV 0.7). 
Liking for contact days is only partially explained by their much-liked content and them providing "concrete teaching." Because learners in T4 and T5 were busy professionals, they felt that at workplace, work was everpresent, taking precedence over studying even though they had a permission to use working time for studying: "...[studying] took the last place because work matters were more important." Consequently, learners felt that contact days allowed them to fully focus on learning: “...you were there, you did that, listened and were part of what was being done, and work didn't bother-it was waiting but it didn't jump on you." Learners ended up doing social media related assignments "Friday afternoons" "when you no longer can focus" or weekends and late evenings. Also, learners felt that some information is hard to transmit unless physically in the same space.

Learning in the workplace presented also other challenges. Colleagues often dropped by to ask something, breaking concentration and, in case of synchronous tools, causing learners to lose the track of the conversation. Even if colleagues did not disturb learners directly, some found background noises in open offices disturbing. Moreover, while colleagues were usually understanding, some learners felt that they were under scrutiny: "...somebody commented that you've been too long on the phone [voice conference]... 'Aren't you working' and that kind of comments..."

In voice conferencing, some learners were uncomfortable with others being able to hear what they said: “.... [it] is related to people around me... you can't talk too freely in that situation." Overall, chat as silent was seen as perhaps better suiting office environment.

While T5 felt that social media tools afforded little interactivity, T4 felt that synchronous tools afforded interactivity and supported e.g. networking, peer support, and sharing of experiences. In addition they gave peer interactions more focus and involved a larger number of peers than contact day impromptu meetings with peers. The important distinction is that T5 had only one chat session while T4 had three chat sessions and two voice conferences. Synchronous tools provided interactivity while asynchronous failed in that.

\section{Synchronous Tools: Chat and Voice Conferencing}

The two synchronous features, chat and voice conferencing (MS Live Meeting), were used in small groups of 4-6 learners. Learners liked both features and felt that they provided social presence, were informal by tone and were close to having a face-to-face conversation, unlike the asynchronous features. Using them was likened to enhanced "corridor conversations" in that they enabled more people to participate and exchanges to have a clear focus. Also, using them was seen as more participatory and active than lecture-based learning.

In effect, synchronous tools were seen as inherently interactive-one constantly reacted and responded to what was being said, with conversation flowing from "intuitive thinking.” The interactive process provided instantaneous and plentiful feedback, experience sharing and "useful ideas." Learners also felt that the process provided peer support and a feeling that others were dealing with similar challenges.

However, both tools presented challenges, too. Chat was seen especially by less experienced learners as too fast-paced, with the conversation moving on while one was typing one's response, leading to conversations overlapping and making following it challenging. However, simultaneously, the few more experienced chatters felt that the conversation was "slow" and "email-like," underlining initial skill differences among the learners. In voice conferencing, the biggest problem was turn-taking. Learners felt unsure when they could speak, which easily led to 2-3 learners dominating the conversation while others listened passively.

While many learners viewed experience-based peer feedback, peer support and sharing of ideas as the best thing about social media tools, some felt that while "fun," it did not represent real learning. They missed "concrete teaching" and suggested that conversations"occasionally...just chitchatting"—should have been based on e.g. readings or other materials to give them substance.

\section{Asynchronous tools: Blog, Discussion forum, and Wiki}

Overall, learners received the asynchronous tools lukewarmly. Learners spoke having “...a feeling that I'm at school..." and felt that they were expected to submit " $a$ well thought-out whole ... a finished article." At best, these tools were found useful in that they forced one to slow down and reflect but were largely seen as a "chore." Asynchronous tools were seen as non-interactive, something done "alone."

Learners were not divided into small groups for asynchronous tools, which led to a "gray mass" of texts "flooding" in close to the deadlines, "numbing" the learners who made few comments (non-compulsory). Some learners felt that postings also in asynchronous tools were "rather superficial” and “didn't bring much deeper knowledge."

Blogs. As few comments were made and the blog "assignments" had one deadline in T4, it ended up a system of submitting assignment. Learners received little feedback from peers or instructors, which discouraged them: "...I started to wonder if it actually interested anybody at all.'

In contrast, in $\mathrm{T} 5$, blogs were introduced as learning diaries and there was no one deadline. Now, even though learners still felt that there was not much interactivity- "it stayed a bit diary-like"-22 out of 61 postings received altogether 36 comments (1-3 per posting). About half of them were by instructors, a quarter by peer learners, and a quarter by authors responding to comments.

Interestingly, while some learners in $\mathrm{T} 5$ felt that "there was no function, no need for writing a blog," some others found it the best social media feature in the training, as it made them "analyze what I have learned," i.e. provided means for introspection on learning. In effect, the learners who intuited the self-reflective use were happy and motivated while those who did not intuit it were not.

Learners in T5 reported feeling that they wrote their diaries more carefully because of knowing that others read them and also tried to make them interesting for others, thus indicating that some level of social presence in fact was present. Also, learners felt that what was written in blogs was different from what they spoke about in face-toface interactions. Finally, learners missed interactivity from blogs but were not motivated to make it happen, as there was no reward for it. 
Discussion forums. In discussion forums, the posting also flowed in close to deadlines, resulting in practically no commenting and desultory readings at best: “...there're just too many messages, it numbs you and then you don't comment anything...” Also, everybody wrote on the same topic, resulting in a very boring read. Overall, few learners commented on forums positively. One learner summed it up by stating that she "spat" her "produce" there "and that was that." Learners felt that posting interested nobody and therefore they simply did the assignments and moved on.

Wikis. Learners invariably saw wiki as a "chore” and felt that it served no function in the training even though they did find existing online wikis (e.g. Wikipedia) very useful. Instructors presented wiki as something to try, which learners found singularly unmotivating. Learners were further unmotivated by the fact that the wiki assignment replicated the information already available in their intranet: "...I don't know if the results are used for anything since we have better descriptions in the intra..."

Although learners understood the idea behind a wiki, they simply got physically together (in small groups they were assigned to) and did the wikis at one or two sittings as group works - despite having difficulties to find suitable times. They felt that expanding or correcting of wiki pages was not expected of them-in fact, they felt that they were not even expected to read the contents that other small groups produced. Wiki ended up being a storage place for the group work results with no interactivity online. In fact, learners did not even see wiki as interactive per se.

\section{HOW TO INSTILL INTERACTIVE LEARNING PROCESS}

Synchronous tools appeared inherently interactive to learners, providing peer support, networking and sharing of experiences in addition to experience-based feedback, ideas and suggestions. Still, while synchronous tools were widely liked, not everybody saw them as providing real learning: "We shared experiences and tips...but I really didn't get much else out of them." Asynchronous tools, in contrast, largely failed to engender interaction and were seen as "chores" done "alone". The only exception was blog when used as a learning diary. The learners who saw its potential for self-reflection on learning liked it while others did not.

These pilots showed that social media tools and approaches can provide value to learners, but the benefits are not automatic. The learning process does not emerge from using social tools but the tools can be used as means to social e-learning. The interactive learning process itself needs to be designed to be continuous and it needs maintaining.

Figure 1 (below) summarizes the most important elements in instilling interactive learning culture in a CPD training.

\section{A. Level skill differences with tool training}

Learners came to their training with varying skills at social media. In T1 and T2, where learners came from a retail organization, some did not even use email daily. Even in T3-T5, where learners hailed from insurance and used computers in daily work, there were also significant skill differences. While some had used social media tools for decades, some others were completely new to the tools. Making assumptions about technical skills is dan- gerous, as not all in T4 knew how to use Live Meeting even though it was installed on everybody's computer as per company policy.

As trainings offered only limited tool training, learners were left to figure them out on their own: “....it was a challenge to study the tool itself before you got to the actual matter." Learners considered building confidence at tools important to enable everybody to participate and contribute at equal footing. Also, skillfulness has been shown to increase both learning and satisfaction [14]. The importance of tool training is further underlined by the fact that even learners with IT background and initial training have had technical proficiency problems [8]. Furthermore, use culture, e.g. style of writing, also needs to be taught as some inexperienced learners were unsure about what to write and in what style.

In addition to providing tool training that brings everybody up to the scratch, timing of the training is important. In T5, wiki training took place at the beginning, and learners complained that by the time they were supposed to start writing wikis, they had forgotten it all. Tool training should take place right before using the tool so that learners immediately put into practice what they have learned.

A good approach is to use the tools the organization makes available to workers. This way learners do not need to install anything, at least some are already skilled and after training learners can continue to use the tools in work.

\section{B. Explicate benefits to engender mindset change}

Explicating the benefits of using social media tools and how to use them to gain those benefits is essential. At least some learners may fail to see such benefits as interaction with peers that engenders experience-based feedback as real learning (see also [11]). Learners want the question "What do I benefit from doing this?" answered. Providing the answer is essential to engender a mindset change that turns learners from passive consumers into active contributors [11] and to motivate learners to participate.

As it was, however, some learners were left confused about the role of the social tools and how to approach learning with such tools: “...we might have gotten a tad off the given topic [in chats] but I don't know if that's a good thing or a bad thing." Learners need guidance as how to reap the benefits from the new approach. Now learners largely felt that all they were supposed to do were the assignments. While commenting was encouraged, it was not compulsory or part of the assessment. Also, learners, while aware that reading the posting of others would be good, felt that it was not encouraged. In T5 learners even felt that they were not expected to read, correct or expand the wikis of others.

This approach appears to have been implicitly encouraged by instructors (see also [2]): “...I wish them to do things and doing is always visible." Instructors used the old model for evaluating learners, leaving learners scant motivation to interact in asynchronous tools. If consumption is not valued, there is no motivation to read the postings, and if postings are not read, there will be no comments. In social media, learners become prosumers [3] and instructors need to understand the implications of this. 


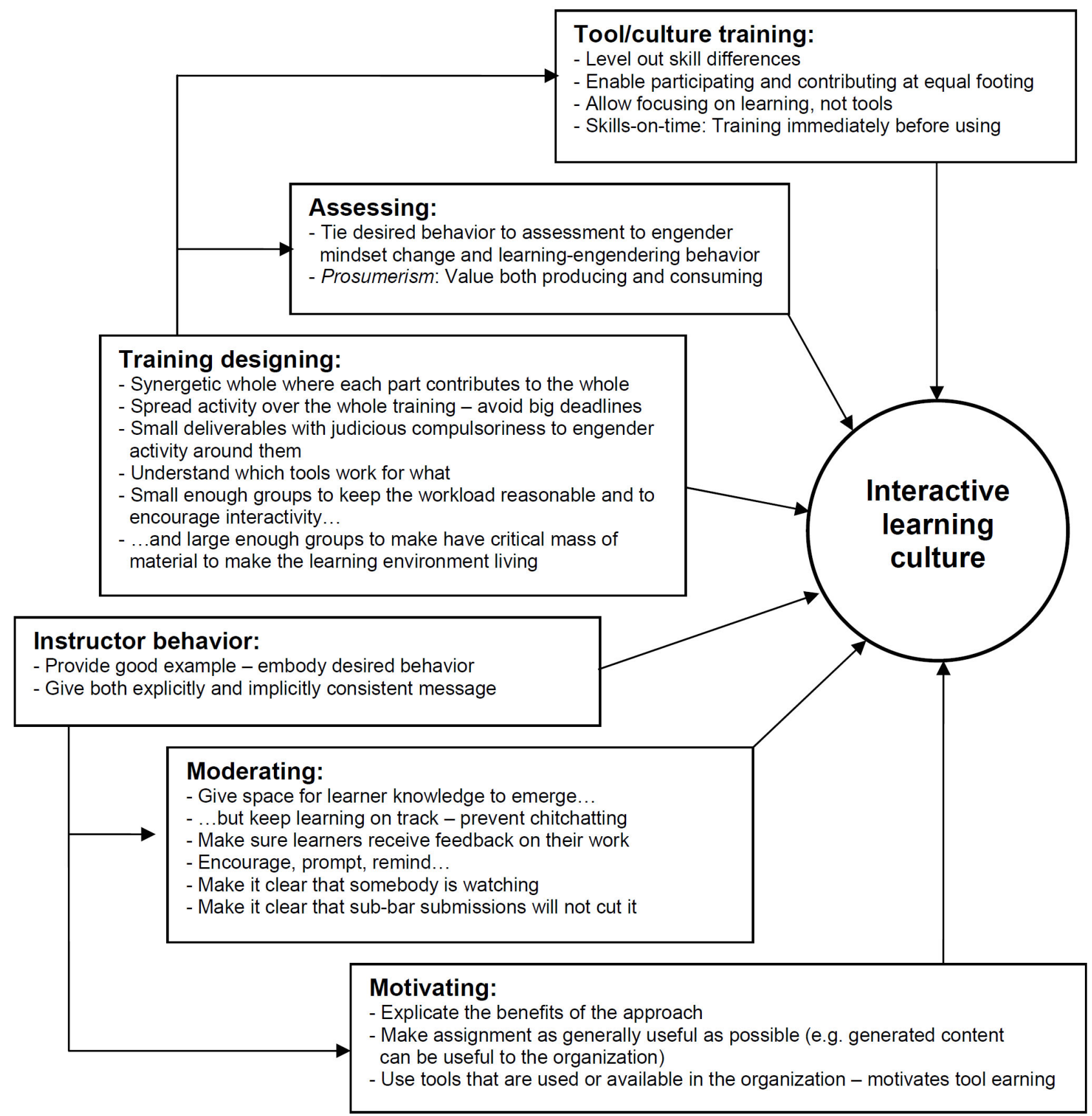

Figure 1. Facilitating the emergence of interactive learning culture: elements of success

Consequently, tying desired behavior to assessment is necessary for both mindset change and showing learners how to gain the promised benefits: Assessing should be based on what engenders learning, guiding learners to engage in interactive learning process (See also [5]). Explicating benefits but not valuing them in assessing will not work.

\section{Design for continuous interaction and maintain it}

Based on earlier research $[1,3,5]$ and experiences from these pilots, trainings need to be designed as synergetic wholes where interactivity is maintained throughout the learning process. With synchronous tools that inherently promote interactivity, the challenge is to integrate them to the whole, to create "a coherent package." In contrast, asynchronous tools are the ones that need to maintain a continuous learning process throughout the training. Moreover, learners wished that using asynchronous tools would have been more interactive instead of them doing "assignments" "alone." Interactivity was seen as having potential to provide feedback that learners now felt was missing.

Consequently, instead of big deadlines that only result in postings "flooding" in close to deadlines and turning the learning space into a "gray mass" of text that discourages learners from reading or commenting, asynchronous tools should employ smaller deadlines spread across the training and reasonable compulsoriness for reading and responding to postings to induce interactivity. If activity is not continuous, learners need to break inertia repeatedly: "When you go there rarely, the threshold of starting is always as high." 
Moreover, compulsoriness and deadlines need to be enforced-learners wished to know that what they do matters and that sub-bar contributions are not acceptable. Learners were aware of their activity being up to them on the last hand but still hoped instructors to provide "encouragement and prompting.” Signs and signals of oversight are important in this: "...tell us right from the scratch that "we will be watching how you are doing there." Learners felt that moderation was "vague" while they wanted to have clear rules: “...clear dates and if I haven't written by then, something to prompt, some kind of sanction or maybe carrot to start it up." Learners also stated that emphasizing good contributions is important to provide examples of what is expected. Also, learners do expect expert feedback from instructors.

In using compulsoriness, reasonableness is the key (see also [1, 14]): If workload is too large, learners are discouraged: “...too many messages, it numbs you and then you don't comment anything,"and if the workload is too light, then "a critical mass" of postings and comments will not be reached for interactivity, and "it simply dies."

Group size is an important consideration here. Different tools may require different small group sizes, e.g. learners suggested 3-4 for chats and ref. [14] posited 6-9 as suitable for blogging. In these pilots, too large group sizes in synchronous tools resulted in some learners having difficulties in participating, while in asynchronous tools not dividing learners to groups resulted in unreasonable amount of work. Small group sizes are also necessary to help everybody participate: “...if the group is too large, people feel too shy or don't dare to participate or don't have time to talk..." If more than one tool is used, it may be necessary to rearrange small groups-or combine them, as e.g. two 4-learner chat groups would conveniently make one 8-learner blog group.

\section{Other motivational factors}

In these pilots, instructors unintentionally ended up undermining learner motivation, something that better designing and example-setting could have avoided. In T5, learners perceived wiki as something they were to try for the sake of trying. Learners were demotivated, as their motivation depends on thing having a function: "Well, the first thing is to consider the function, purpose, reason why ...that we are just testing is not very motivating." Furthermore, learners perceived the wiki assignment as duplicating information available in their intranet, a case of bad designing that led to reducing learner motivation.

Likewise, in T4, when learners received no feedback on their blog assignments, they felt that "it all stayed hanging in the air" while they could "have refined them and turned the material into something that would have stayed as ... instructional material." Producing something that has no clear or further purpose was clearly frustrating to learners. At the very least, learners need to get feedback on their work to see it as worthwhile. Finally, instructors need to set good example. In T5, learners felt that the instructors' blog was inactive and consequently set a bad example.

\section{CONCLUSIONS}

Our results add to the growing body of evidence that while social media tools can be used in e-learning and they can bring value to CPD trainings, the process does not emerge automatically from adding the tools-tools are not inherently collaborative [5] — but needs to be designed and maintained.

Instilling interactive learning culture depends on a mindset change [12] and rests on learner motivation. For mindset change to take place, learners need to understand the value and the benefits that social e-learning can offer in addition to seeing how to gain those benefits. Motivating learners also requires the training to constitute a synergetic whole where each part clearly contributes to the whole. As asynchronous tools need to maintain the learning process between synchronous-tool sessions and contact teaching days, assignments need to be divided into smaller deliverables and reasonable compulsoriness appears necessary to maintain interactivity around the deliverables to provide a constant flow of feedback. As with synchronous tools, moderation also plays a significant role in asynchronous tools. Instructors need to maintain activity and promote high-quality contributions. Furthermore, assignments need to make sense to learners, and ideally the results should have uses beyond the immediate learning goals to motivate busy workplace learners.

Regrettably, our results also show that the early research findings have not filtered over to practitioners, training designers and instructors, as many mistakes are being repeated (see also [9]). We encourage reaching out to the practitioner community and attending their conferences and events to disseminate the results of the ongoing research effort. Lest we do, "misunderstandings, disappointments and irrelevant pedagogical practices" [9] continue to plague the field.

\section{REFERENCES}

[1] M. Cole, "Using Wiki Technology to Support Student Engagement: Lessons from the Trenches,” Computers \& Education, vol. 52, pp. 141-146, 2009. http://dx.doi.org/10.1016/j.compedu.2008. $\underline{07.003}$

[2] N.B. Dohn, "Teaching with Wikis and Blogs: Potentials and Pitfalls,” Proc. 7th Int. Conference on Networked Learning, pp. 142-150, 2010.

[3] C. McLoughlin and M.J.W. Lee, "Social Software and Participatory Learning: Pedagogical Choices with Technology Affordances in the Web 2.0 Era,” In: Proc. Ascilite'07, pp. 664-675, 2007.

[4] D.H. Spencer and S.R. Hiltz, "A Field Study of Use of Synchronous Chat in Online Courses,” Proc. 36th Hawaii Int. Conference on System Sciences, pp. 1-10, 2003. http://dx.doi.org/10.1109/ HICSS.2003.1173742

[5] T. Judd, G. Kennedy, and S. Cropper, "Using Wikis for Collaborative Learning: Assessing Collaboration through Contribution," AJET, vol. 26(3), pp. 341-354, 2010.

[6] M. Zenios and B. Holmes, "Knowledge Creation in Networked Learning: Combined Tools and Affordances,” Proc. 7th Int. Conference on Networked Learning, pp. 471-479, 2010.

[7] L. Lockyer, and J. Patterson, “Integrating Social Networking Technologies in Education: A Case Study of a Formal Learning Environment," Proc. 8th Int. Conference on Advanced Learning Technologies, pp. 529-533, 2008. http://dx.doi.org/10.1109/ ICALT.2008.67

[8] S.O. Choy and K.C. Ng, “Implementing Wiki Software for Supplementing Online Learning," AJET, vol. 23(2), pp. 209-226, 2007.

[9] P. Tynjälä and P. Häkkinen,”E-learning at Work: Theoretical Underpinnings and Pedagogical Challenges," Journal of Workplace Learning, 17(5/6), pp. 318-336, 2005. http://dx.doi.org/10.1108/13665620510606742

[10] F. Daneshgar, C. Van Toorn, S.C.E. Chan, "E-learning in Workplaces," Proc. ITI 6th Int. Conference on Information \& Communications Technology, pp. 65-70, 2008. 
[11] G. Kirkpatrick, "Online 'Chat' Facilities as Pedagogic Tools: A Case Study,” AHL, vol. 6, pp. 145-159, 2005.

[12] G. Fischer, "Lifelong Learning and Its Support with New Media," in Int. Encyclopedia of Social and Behavioral Sciences, vol. 13, N.J. Smelser and P.B. Baltes, Eds., Elsevier, 2001, pp. 8836-8840. http://dx.doi.org/10.1016/B0-08-043076-7/04675-1

[13] A. Ruth and L. Houghton, "The Wiki Way of Learning," AJET, vol. 25(2), pp. 135-152, 2009.

[14] R.K. Ladyshewsky and P. Gardner, "Peer Assisted Learning and Blogging: A Strategy to Promote Reflective Practice during Clinical Fieldwork,” AJET, 24(3), pp. 241-257, 2008.

\section{AUTHORS}

Juha Leino, Erika Tanhua-Piiroinen, and Johanna Sommers-Piiroinen are with the University of Tampere, SIS/TRIM, 33014 Tampereen yliopisto, Finland (e-mail: firstname.lastname@uta.fi).

This article is an extended version of a paper presented at the conference ICELW2012, held June 2012, at Columbia University, in New York, NY, USA. Manuscript received 11th July 2012. Published as resubmitted by the authors 5 August 2012. This work was supported in part by the Finnish Funding Agency for Technology and Innovation, TEKES. 